\section{Des mutations \\ de la dynamine 2 à l'origine de la forme dominante de la myopathie centronucléaire}

Marc Bitoun, Norma B. Romero, Pascale Guicheney
Inserm U.582,

Université Pierre et Marie Curie, AP-HP, IFR 14, CHU Pitié-Salpêtrière, 105, boulevard de l'Hôpital, 75013 Paris, France. p.guicheney@myologie.chups.jussieu.fr
> Les myopathies centronucléaires (MCN) constituent un groupe de myopathies congénitales cliniquement et génétiquement hétérogène affectant moins d'une personne sur 10000 . Depuis la première description de la pathologie en 1966 [1], plusieurs formes de MCN ont été individualisées en fonction de leur transmission génétique: la myo-

pathie myotubulaire qui est la forme la plus sévère liée au chromosome $X$ et les formes autosomiques (dominante et récessive) cliniquement moins graves. Des mutations responsables de la myopathie myotubulaire ont été identifiées dans le gène codant la myotubularine (MTMI), une phospho-inositide phosphatase potentiellement impliquée dans

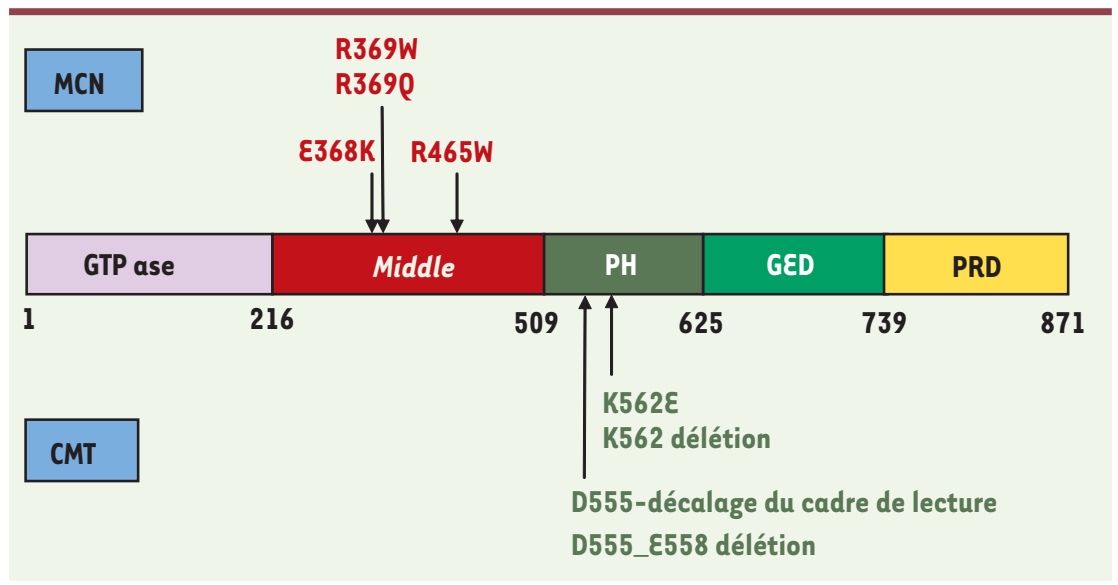

Figure 1. Représentation des domaines de la DNM2 et positionnement des mutations identifiées dans la myopathie centronucléaire et la maladie de Charcot-Marie-Tooth. La dynamine 2 est une protéine multi-domaine constituée d'un domaine GTPase amino-terminal responsable de la liaison et de l'hydrolyse du GTP, d'un domaine intermédiaire (Middle) sans motif caractéristique, d'un domaine d'homologie à la pleckstrine $(P H)$ responsable de sa liaison aux phospho-inositides membranaires, d'un domaine GED (GTPase effector domain) régulant son activité GTPasique et d'un domaine PRD (proline rich domain) carboxy-terminal d'interaction avec le domaine SH3 de protéines partenaires. Les mutations identifiées dans la myopathie centronucléaire (MCN) sont jusqu'à présent toutes des mutations faux sens, restreintes au domaine intermédiaire. Les mutations identifiées dans la maladie de Charcot-Marie-Tooth (CMT) sont restreintes au domaine PH et incluent une mutation faux sens, une délétion de l'acide aminé 562 et une délétion de 9 paires de bases à l'origine de 2 protéines différentes: soit une délétion de 3 acides aminés, soit un décalage du cadre de lecture conduisant à une protéine tronquée par insertion d'un codon stop prématuré. Les acides aminés sont indiqués en symbole international d'une lettre. La numérotation des acides aminés est déterminée sur la séquence protéique humaine de Genbank P50570. des processus de trafic membranaire [2]. En revanche, l'origine génétique des formes autosomiques était encore indéterminée. Les patients atteints de la forme autosomique dominante de MCN présentent une faiblesse musculaire lentement progressive le plus souvent associée à une atteinte oculaire entraînant un ptosis ou une ophtalmoplégie [3]. L'exploration des biopsies musculaires permet de diagnostiquer cette forme de myopathie congénitale par l'observation d'une proportion élevée de fibres musculaires contenant des noyaux centralisés, alors que les noyaux sont en position périphérique dans les fibres musculaires matures normales, en association avec une prédominance des fibres de type I et la présence de travées sarcoplasmiques irradiant des noyaux centraux, donnant à ces fibres un aspect caractéristique en «rayon de roue».

Une approche de génétique inverse a été menée afin d'identifier le gène dont les mutations sont responsables de la forme autosomique dominante de la MCN. Le génotypage de marqueurs microsatellites dans trois familles a mis en évidence une liaison génétique entre la MCN et un locus commun aux trois familles situé sur le bras court du chromosome 19 en position 19p13.2. Par cette approche, ce locus a été restreint à un intervalle de 11 mégabases contenant environ 200 gènes. Le criblage de gènes candidats présents dans cette région du génome a conduit à l'identification de mutations faux sens dans la séquence du gène 
codant la dynamine 2 (DNM2), provoquant la substitution d'un acide aminé par un autre dans la séquence de la protéine. Quatre mutations différentes ont été trouvées chez 50 patients (issus de 11 familles, atteints de la forme autosomique dominante, ou de cas sporadiques) [4].

Les dynamines sont des GTPases de haut poids moléculaire (environ 100 kDa) impliquées dans le trafic membranaire. Ces molécules hydrolysent le GTP et participent à la déformation des membranes biologiques nécessaire à la formation de vésicules [5]. Parmi les trois dynamines que compte cette famille chez l'homme, la dynamine 2 est une protéine ubiquitaire principalement impliquée dans l'endocytose et le trafic membranaire intracellulaire ainsi que dans la dynamique du réseau d'actine et la structure du centrosome. Les quatre mutations que nous avons identifiées sont restreintes à un domaine protéique nommé domaine intermédiaire (middle domain) adjacent au domaine d'homologie à la pleckstrine (Figure 1) où des mutations ont récemment été identifiées dans la forme autosomique dominante intermédiaire de la maladie de Charcot-Marie-Tooth [6]. L'implication du domaine intermédiaire dans la régulation de l'activité GTPasique et dans l'auto-assemblage de la dynamine 2 a été rapportée. Plus récemment, son rôle dans la localisation centrosomique de la dynamine 2 a également été démontré [7]. Afin de tester l'impact des mutations de la DNM2 sur la localisation au centrosome de cette protéine, nous avons produit des vecteurs plasmidiques permettant leur expression in vitro. Cette approche nous a permis de montrer une réduction de la localisation centrosomale des mutants de la DNM2 [4]. Nos hypothèses actuelles concernant la physiopathologie de la MCN formulent que les mutations de la DNM2 induisent une altération du trafic membranaire et interfèreraient avec la fonction de protéines du centrosome.

\section{Conclusions et perspectives}

Notre étude rapporte la première identification d'un gène muté dans la forme autosomique dominante de la myopathie centronucléaire. Nos résultats ouvrent la voie vers la compréhension de la physiopathologie de cette myopathie à travers la détermination des dysfonctionnements cellulaires engendrés par ces mutations qui permettra le développement futur de stratégies thérapeutiques. $\diamond$

Mutations in dynamin 2 cause

dominant centronuclear myopathy

\section{RÉFÉRENCES}

1. Spiro AJ, Shy GM, Gonatas NK. Myotubular myopathy. Persistence of fetal muscle in an adolescent boy. Arch Neurol 1966; 14 : 1-14.

2. Laporte J, Hu LJ, Kretz C, et al. A gene mutated in $X$-linked myotubular myopathy defines a new putative tyrosine phosphatase family conserved in yeast. Nat Genet 1996; $13: 175-82$.

3. Jeannet Py, Bassez G, Eymard B, et al. Clinical and histologic findings in autosomal centronuclear myopathy. Neurology 2004 ; 62 : 1484-90.

4. Bitoun, M. Maugenre S, Jeannet Py, et al. Mutations in dynamin 2 cause dominant centronuclear myopathy. Nat Genet 2005; 37 : 1207-9.

5. Praefcke GJ, McMahon HT. The dynamin superfamily : universal membrane tubulation and fission molecules? Nat Rev Mol Cell Biol 2004 ; 5 : 133-47.

6. Züchner S, Noureddine M, Kennerson, et al. Mutations in the pleckstrin homology domain of dynamin 2 cause dominant intermediate CharcotMarie-Tooth disease. Nat Genet 2005 ; 37 : 289-94.

7. Thompson H, Cao H, Chen J, et al. Dynamin 2 binds gamma-tubulin and participates in centrosome cohesion. Nat Cell Biol $2004 ; 6$ : 335-42.

\section{NOUVELLE}

\section{La souris paraplégique \\ Un modèle offrant de nouvelles perspectives de recherche sur les lésions de la moelle épinière}

Nicolas P. Lapointe, Pierre A. Guertin

\author{
Département d'anatomie \\ et de physiologie, \\ Unité de neuroscience, \\ RC-98002705, \\ Centre Hospitalier de l'Université Laval, \\ boulevard Laurier, Québec GIV 4G2, Canada. \\ Pierre.Guertin@crchul.ulaval.ca
}

> Plus de 500000 patients souffrant d'une lésion à la moelle épinière sont recensés en Amérique du Nord et en Europe. Les dommages causés par la lésion chez ces personnes sont généralement dévastateurs. En effet, les processus régénératifs spontanés de la moelle épinière sont presque inexistants et, à ce jour, aucun traitement n'a permis la réparation des tissus lésés et le rétablissement des con- nexions avec le cerveau. Une lésion spinale entraîne généralement la perte immédiate, totale ou partielle, de sensations et de contrôles moteur et locomoteur volontaire sous le niveau lésé conduisant à des problèmes d'atrophie musculaire, d'ostéoporose, de déficiences du système immunitaire et cardiovasculaire souvent accompagnées d'infections cutanées, rénales et pulmonaires.
Des études classiques réalisées il y a plus d'une quinzaine d'années avaient mis en évidence la capacité qu'ont certains agents sérotoninergiques de favoriser la récupération des fonctions locomotrices. $\varepsilon n$ effet, chez des chats paraplégiques, des précurseurs et agonistes de la sérotonine (5-hydroxytriptamine [5-HT]) tels que le 5-hydroxytryptophane et la quipazine augmentent la contraction muscu- 